\title{
ON INJECTIVE RESOLUTIONS OF LOCAL COHOMOLOGY MODULES
}

\author{
TONY J. PUTHENPURAKAL
}

\begin{abstract}
Let $K$ be a field of characteristic zero and let $R=K\left[X_{1}, \ldots, X_{n}\right]$. Let $I$ be an ideal in $R$ and let $M=H_{I}^{i}(R)$ be the $i^{t h}$-local cohomology module of $R$ with respect to $I$. Let $c=\operatorname{injdim} M$. We prove that if $P$ is a prime ideal in $R$ with Bass number $\mu_{c}(P, M)>0$ then $P$ is a maximal ideal in $R$.
\end{abstract}

\section{INTRODUCTION}

Throughout this paper $R$ is a commutative Noetherian ring. If $M$ is an $R$-module and $Y$ be a locally closed subscheme of $\operatorname{Spec}(R)$, we denote by $H_{Y}^{i}(M)$ the $i^{t h}$-local cohomology module of $M$ with support in $Y$. If $Y$ is closed in $\operatorname{Spec}(R)$ with defining ideal $I$ then $H_{Y}^{i}(M)$ is denoted by $H_{I}^{i}(M)$.

In a remarkable paper, 4, Huneke and Sharp proved that if $R$ is a regular ring containing a field of characteristic $p>0$, and $I$ is an ideal in $R$ then the local cohomology modules of $R$ with respect to $I$ have the following properties:

(i) $H_{\mathfrak{m}}^{j}\left(H_{I}^{i}(R)\right)$ is injective, where $\mathfrak{m}$ is any maximal ideal of $R$.

(ii) $\operatorname{injdim}_{R} H_{I}^{i}(R) \leq \operatorname{dim} \operatorname{Supp} H_{I}^{i}(R)$.

(iii) The set of associated primes of $H_{I}^{i}(R)$ is finite.

(iv) All the Bass numbers of $H_{I}^{i}(R)$ are finite.

Here $\operatorname{inj}_{\operatorname{dim}} H_{I}^{i}(R)$ denotes the injective dimension of $H_{I}^{i}(R)$. Also Supp $M=$ $\left\{P \mid M_{P} \neq 0\right.$ and $P$ is a prime in $\left.R\right\}$ is the support of an $R$-module $M$. The $j^{t h}$ Bass number of an $R$-module $M$ with respect to a prime ideal $P$ is defined as $\mu_{j}(P, M)=\operatorname{dim}_{k(P)} \operatorname{Ext}_{R_{P}}^{j}\left(k(P), M_{P}\right)$ where $k(P)$ is the residue field of $R_{P}$.

In another remarkable paper, for regular rings in characteristic zero, Lyubeznik was able to establish the above properties for a considerably larger class of functors than just the local cohomology modules, see [5]. We call such functors as Lyubeznik functors, see section two for details. If $\mathcal{T}$ is a Lyubeznik functor on $\operatorname{Mod}(R)$ then $\mathcal{T}(R)$ satisfies the following properties:

(i) $H_{\mathfrak{m}}^{j}(\mathcal{T}(R))$ is injective, where $\mathfrak{m}$ is any maximal ideal of $R$.

(ii) $\operatorname{injdim}_{R} \mathcal{T}(R) \leq \operatorname{dim} \operatorname{Supp} \mathcal{T}(R)$.

(iii) For every maximal ideal $\mathfrak{m}$, the number of associated primes of $\mathcal{T}(R)$ contained in $\mathfrak{m}$ is finite.

(iv) All the Bass numbers of $\mathcal{T}(R)$ are finite.

We should note that if $R=K\left[X_{1}, \ldots, X_{n}\right]$ then the number of associate primes of $\mathcal{T}(R)$ is finite.

Date: January 17, 2020.

1991 Mathematics Subject Classification. Primary 13D45; Secondary 13D02, 13H10 .

Key words and phrases. local cohomology, injective resolutions. 
The results of Lyubeznik for characteristic zero raised the question of whether the results (i)-(iv) of Huneke and Sharp (in characteristic $p>0$ ) could be extended to this larger class of functors. In [6], Lyubeznik proves it.

If $M$ is a finitely generated module over a Cohen-Macaulay $\operatorname{ring} R$ and say $M$ has finite injective dimension $d=\operatorname{dim} R$, then it is elementary to prove that if $\mu_{d}(P, M)>0$ then $P$ is a maximal ideal in $R$, use [3, 3.1.13]. This fails for modules which are not finitely generated, for instance consider the injective hull $E(R / P)$ of $R / P$ where $P$ is a prime ideal which is not maximal.

Our main result is

Theorem 1.1. Let $K$ be a field of characteristic zero and let $R=K\left[X_{1}, \ldots, X_{n}\right]$. Let $\mathcal{T}$ be a Lyubeznik functor on $\operatorname{Mod}(R)$. Suppose $\operatorname{inj\operatorname {dim}} \mathcal{T}(R)=$ c. If $P$ is a prime ideal in $R$ with Bass number $\mu_{c}(P, \mathcal{T}(R))>0$ then $P$ is a maximal ideal of $R$.

As an aside we note that to best of our knowledge this is the first result whose proof uses the fact that $\operatorname{Ass} \mathcal{T}(R)$ is finite for any Lyubeznik functor $\mathcal{T}$.

A natural question is what can we say about $\mu_{c}(\mathfrak{m}, \mathcal{T}(R))$ as $\mathfrak{m}$ varies over maximal ideals in $R$. Our next result is essentially only an observation.

Proposition 1.2. Let $K$ be an algebraically closed field of characteristic zero and let $R=K\left[X_{1}, \ldots, X_{n}\right]$. Let $\mathcal{T}$ be a Lyubeznik functor on $\operatorname{Mod}(R)$. Suppose $\operatorname{inj\operatorname {dim}} \mathcal{T}(R)=c$. Then for all $i=0, \ldots, c$; the set

$$
\left\{\mu_{i}(\mathfrak{m}, \mathcal{T}(R)) \mid \mathfrak{m} \text { a maximal ideal of } R\right\}
$$

is bounded.

The surprising thing about Proposition 1.2 is that I do not know whether such a result holds for finitely generated modules over $R$.

A natural question is whether the results 1.1 and 1.2 hold in characteristic $p>$ 0 . Although we expect this to be true; our techniques do not work in positive characteristic. We are only able to extend Propostion 1.2 to a subclass of Lyubeznik functors, see 5.3

We now describe in brief the contents of this paper. In section two we define Lyubeznik functors and also a few preliminary results on holonomic modules which we need. In section three we discuss two Lemmas which will help in proving Theorem 1.1. In section four we prove Theorem 1.1. Finally in section five we prove Proposition 1.2

\section{Preliminaries}

In this section we define Lyubeznik functors. We also prove a result on holonomic modules which we need.

2.1. Lyubeznik functors:

Let $R$ be a commutative Noetherian ring and let $X=\operatorname{Spec}(R)$. Let $Y$ be a locally closed subset of $X$. If $M$ is an $R$-module and $Y$ be a locally closed subscheme of $\operatorname{Spec}(R)$, we denote by $H_{Y}^{i}(M)$ the $i^{\text {th }}$-local cohomology module of $M$ with support in $Y$. Suppose $Y=Y_{1} \backslash Y_{2}$ where $Y_{2} \subseteq Y_{1}$ are two closed subsets of $X$ then we have an exact sequence of functors

$$
\cdots \rightarrow H_{Y_{1}}^{i}(-) \rightarrow H_{Y_{2}}^{i}(-) \rightarrow H_{Y}^{i}(-) \rightarrow H_{Y_{1}}^{i+1}(-) \rightarrow
$$


A Lyubeznik functor $\mathcal{T}$ is any functor of the form $\mathcal{T}=\mathcal{T}_{1} \circ \mathcal{T}_{2} \circ \cdots \circ \mathcal{T}_{m}$ where every functor $\mathcal{T}_{j}$ is either $H_{Y}^{i}(-)$ for some locally closed subset of $X$ or the kernel, image or cokernel of some arrow in the previous long exact sequence for closed subsets $Y_{1}, Y_{2}$ of $X$ such that $Y_{2} \subseteq Y_{1}$.

We need the following result from [5, 3.1].

Proposition 2.2. Let $\phi: R \rightarrow S$ be a flat homomorphism of Noetherian rings. Let $\mathcal{T}$ be a Lyubeznik functor on $\operatorname{Mod}(R)$. Then there exists a Lyubeznik functor $\widehat{\mathcal{T}}$ on $\operatorname{Mod}(S)$ and isomorphisms $\widehat{\mathcal{T}}\left(M \otimes_{R} S\right) \cong \mathcal{T}(M) \otimes_{R} S$ which is functorial in $M$.

2.3. Lyubeznik functors and holonomicity:

Let $K$ be a field of characteristic zero. Let $S=K\left[\left[X_{1}, \ldots, X_{n}\right]\right]$. Let $D$ be the ring of $K$-linear differential operators on $S$. Let $\mathcal{T}$ be a Lyubeznik functor on $\operatorname{Mod}(S)$. If $M$ is any holonomic $D$-module then $\mathcal{T}(M)$ is a holonomic $D$-module; see [5, 2.2d]. In particular $\mathcal{T}(S)$ is a holonomic $D$-module.

Let $R=K\left[X_{1}, \ldots, X_{n}\right]$ and let $A_{n}(K)$ be the $n^{\text {th }}$-Weyl algebra over $K$. Let $\mathcal{T}$ be a Lyubeznik functor on $\operatorname{Mod}(R)$. If $M$ is any holonomic $A_{n}(K)$-module then $\mathcal{T}(M)$ is a holonomic $A_{n}(K)$-module; (the proof in [5, 2.2d] can be modified to prove this result). In particular $\mathcal{T}(R)$ is a holonomic $A_{n}(K)$-module.

Remark 2.4. In 2] holonomic $A_{n}(K)$-modules are called modules belonging to the Bernstein class.

2.5. Let $k$ be a field of characteristic zero and let $S=k\left[\left[Y_{1}, \ldots, Y_{n}\right]\right]$. Let $D$ be the ring of $k$-linear differential operators on $S$. Let $C$ be a simple holonomic $D$-module. Notice $\operatorname{Ass}_{S} C=\{P\}$ for some prime $P$ in $S$. Also $C$ is $P$-torsion; see 2, 3.3.16-17]. It follows from [2, p. 109, lines 3-6] that there exists $h \in(S / P)$ non-zero such that $\operatorname{Hom}_{S}(S / P, C)_{h}$ is a finitely generated $(S / P)_{h}$ module. Let $g$ be a pre-image of $h$ in $S$. Then clearly $\operatorname{Hom}_{S}(S / P, C)_{g}$ is a finitely generated $S_{g}$-module. We now generalize this result.

Proposition 2.6. (with hypotheses as in 2.5) Let $M$ be a holonomic D-module. Assume $\operatorname{Ass}_{S} M=\{P\}$ and $M$ is P-torsion. Then there exists $h \in S \backslash P$ such that $\operatorname{Hom}_{S}(S / P, M)_{h}$ is finitely generated as a $S_{h}$-module.

Proof. Let $0=M_{0} \varsubsetneqq M_{1} \varsubsetneqq M_{2} \varsubsetneqq \cdots \varsubsetneqq M_{n-1} \varsubsetneqq M_{n}=M$ be a filtration of $M$ with $M_{i} / M_{i-1}$ simple $D$-module for $i=1, \ldots, n$. By induction on $i$ we prove that there exists $h_{i} \in S \backslash P$ such that $\operatorname{Hom}_{S}\left(S / P, M_{i}\right)_{h_{i}}$ is finitely generated as a $S_{h_{i}}$-module.

For $i=1$ note that $M_{1}$ is a simple holonomic $D$-module. Also $\operatorname{Ass}_{S} M_{1} \subseteq$ $\operatorname{Ass}_{S} M=\{P\}$. Then by 2.5 we get the required assertion. We assume the result for $i=r$ and prove it for $i=r+1$. Say $\operatorname{Hom}_{S}\left(S / P, M_{r}\right)_{h_{r}}$ is a finitely generated $S_{h_{r}}$-module. We consider the following two cases.

Case 1: $\operatorname{Ass}_{S} M_{r+1} / M_{r}=\{P\}$.

By 2.5 there exists $g_{r} \in S \backslash P$ such that $\operatorname{Hom}_{S}\left(S / P, M_{r+1} / M_{r}\right)_{g_{r}}$ is finitely generated $S_{g_{r}}$-module. Consider the exact sequence

$$
0 \rightarrow \operatorname{Hom}_{S}\left(S / P, M_{r}\right) \rightarrow \operatorname{Hom}_{S}\left(S / P, M_{r+1}\right) \rightarrow \operatorname{Hom}_{S}\left(S / P, M_{r+1} / M_{r}\right) .
$$

Localize at $h_{r+1}=h_{r} g_{r} \in S \backslash P$. Notice

(1) $\operatorname{Hom}_{S}\left(S / P, M_{r}\right)_{h_{r+1}}=\left(\operatorname{Hom}_{S}\left(S / P, M_{r}\right)_{h_{r}}\right)_{g_{r}}$ is finitely generated as a $S_{h_{r+1}}$-module.

(2) $\operatorname{Hom}_{S}\left(S / P, M_{r+1} / M_{r}\right)_{h_{r+1}}=\left(\operatorname{Hom}_{S}\left(S / P, M_{r+1} / M_{r}\right)_{g_{r}}\right)_{h_{r}}$ is finitely generated as a $S_{h_{r+1}}$-module. 
It follows that $\operatorname{Hom}_{S}\left(S / P, M_{r+1}\right)_{h_{r+1}}$ is finitely generated as a $S_{h_{r+1}}$-module.

Case 2: $\operatorname{Ass}_{S} M_{r+1} / M_{r}=\{Q\}$ with $Q \neq P$.

As $M$ is $P$-torsion we have that $Q \supsetneq P$. Take $g \in Q \backslash P$. Then $\left(M_{r+1} / M_{r}\right)_{g}=0$.

So $\operatorname{Hom}_{S}\left(S / P, M_{r+1} / M_{r}\right)_{g}=0$. Put $h_{r+1}=h_{r} g \in S \backslash P$. Then note that

$$
\operatorname{Hom}_{S}\left(S / P, M_{r+1}\right)_{h_{r+1}} \cong \operatorname{Hom}_{S}\left(S / P, M_{r}\right)_{h_{r+1}}=\left(\operatorname{Hom}_{S}\left(S / P, M_{r}\right)_{h_{r}}\right)_{g},
$$

is finitely generated as a $S_{h_{r+1}}$-module. Thus by induction we get that there exists $h \in S \backslash P$ such that $\operatorname{Hom}_{S}(S / P, M)_{h}$ is finitely generated as a $S_{h}$-module.

2.7. Finally we need the following well-known result regarding non-singular locus of affine domains.

Theorem 2.8. Let $A$ be an affine domain, finitely generated over a perfect field $k$. Then

(1) The non-singular locus of $A$ is non-empty and an open subset of $\operatorname{Spec}(A)$.

(2) There exists a maximal ideal $\mathfrak{m}$ of $A$ with $A_{\mathfrak{m}}$ regular local.

(3) If $\operatorname{dim} A \geq 1$ then there exists infinitely many maximal ideals of $A$ with $A_{\mathfrak{m}}$ regular local.

(4) Suppose $\operatorname{dim} A \geq 2$ and let $f \in A$. Then there exists a maximal ideal $\mathfrak{m}$ of $A$ with $f \notin \mathfrak{m}$ and $A_{\mathfrak{m}}$-regular local.

\section{Two Lemma's}

In this section we establish two lemma's which will enable us to prove our main result. Let $K$ be a field of characteristic zero and let $P$ be a prime ideal of height in $R=K\left[X_{1}, \ldots, X_{n}\right]$. Let $E(R / P)$ denote the injective hull of $R / P$. Recall that $E(R / P)=H_{P}^{g}(R)_{P}$. It follows that $E(R / P)$ is a $A_{n}(K)$-module and the natural inclusion $H_{P}^{g}(R) \rightarrow E(R / P)$ is $A_{n}(K)$-linear.

Lemma 3.1. Let $K$ be a field of characteristic zero and let $P$ be a prime ideal of height $n-1$ in $R=K\left[X_{1}, \ldots, X_{n}\right]$. Then $E(R / P)$ is not a holonomic $A_{n}(K)$ module.

Proof. Suppose if possible $E(R / P)$ is a holonomic $A_{n}(K)$-module. We have an exact sequence of $A_{n}(K)$-modules

$$
0 \rightarrow H_{P}^{n-1}(R) \rightarrow E(R / P) \rightarrow C \rightarrow 0 .
$$

As $E(R / P)$ is holonomic we have that $C$ is also a holonomic $A_{n}(K)$-module. Notice $C_{P}=0$. It follows that $C$ is supported at only finitely many maximal ideals of $R$, say $\mathfrak{m}_{1}, \ldots, \mathfrak{m}_{r}$. By Theorem $2.8(3)$ there exists a maximal ideal $\mathfrak{m}$ of $R$ such that $\mathfrak{m} \neq \mathfrak{m}_{i}$ for all $i$ and $(R / P)_{\mathfrak{m}}$ is regular local. Note $H_{P}^{n-1}(R)_{\mathfrak{m}}=E(R / P)_{\mathfrak{m}}$ as $C_{\mathfrak{m}}=0$. If $\mathfrak{m} R_{\mathfrak{m}}=\left(z_{1}, \ldots, z_{n}\right)$ then as $R_{\mathfrak{m}} / P R_{\mathfrak{m}}$ is regular we may assume that $P R_{\mathfrak{m}}=\left(z_{1}, \ldots, z_{n-1}\right)$. In particular $H_{P R_{\mathfrak{m}}}^{n}\left(R_{\mathfrak{m}}\right)=0$.

Let $f \in \mathfrak{m} R_{\mathfrak{m}} \backslash P R_{\mathfrak{m}}$. Note that we have an exact sequence

$$
0 \rightarrow H_{P R_{\mathfrak{m}}}^{n-1}\left(R_{\mathfrak{m}}\right) \rightarrow H_{P R_{\mathfrak{m}}}^{n-1}\left(R_{\mathfrak{m}}\right)_{f} \rightarrow H_{\left(P R_{\mathfrak{m}}, f\right)}^{n}\left(R_{\mathfrak{m}}\right) \rightarrow H_{P R_{\mathfrak{m}}}^{n}\left(R_{\mathfrak{m}}\right)=0
$$

As $H_{P R_{\mathfrak{m}}}^{n-1}\left(R_{\mathfrak{m}}\right)=H_{P}^{n-1}(R)_{\mathfrak{m}}=E(R / P)_{\mathfrak{m}}$ it follows that the first map in the above exact sequence is an isomorphism. It follows that $H_{\left(P R_{\mathfrak{m}}, f\right)}^{n}\left(R_{\mathfrak{m}}\right)=0$. This contradicts Grothendieck's non-vanishing theorem as $\sqrt{\left(P R_{\mathfrak{m}}, f\right)}=\mathfrak{m} R_{\mathfrak{m}}$.

Our next result is 
Lemma 3.2. Let $K$ be a field of characteristic zero and let $P$ be a height $g$ prime in $R=K\left[X_{1}, \ldots, X_{n}\right]$ with $g \leq n-2$. Suppose $\mathfrak{m}$ is a maximal ideal in $R$ with $(R / P)_{\mathfrak{m}}$ a regular local ring. Let $\mathcal{T}$ be a Lyubeznik functor on $\operatorname{Mod}\left(R_{\mathfrak{m}}\right)$. Then $\mathcal{T}\left(R_{\mathfrak{m}}\right) \neq E(R / P)_{\mathfrak{m}}^{c}$ for any $c>0$.

Proof. Suppose if possible $\mathcal{T}\left(R_{\mathfrak{m}}\right)=E(R / P)_{\mathfrak{m}}^{c}$ for some $c>0$. Let $\widehat{R_{\mathfrak{m}}}$ be the completion of $R_{\mathfrak{m}}$ at $\mathfrak{m} R_{\mathfrak{m}}$. Note $\widehat{R_{\mathfrak{m}}}=K^{\prime}\left[\left[Z_{1}, \ldots, Z_{n}\right]\right]$ where $K^{\prime} \cong R / \mathfrak{m}$. Let $D$ be the ring of $K^{\prime}$-linear differential operators on $\widehat{R_{\mathfrak{m}}}$. Note by 2.2 there exists a Lyubeznik functor $\widehat{\mathcal{T}}$ on $\operatorname{Mod}\left(\widehat{R_{\mathfrak{m}}}\right)$ such that $\widehat{\mathcal{T}}\left(\widehat{R_{\mathfrak{m}}}\right)=\mathcal{T}\left(R_{\mathfrak{m}}\right) \otimes \widehat{R_{\mathfrak{m}}}$. In particular $E(R / P)_{\mathfrak{m}}^{c} \otimes \widehat{R_{\mathfrak{m}}}$ is a holonomic $D$-module. So $V=E(R / P)_{\mathfrak{m}} \otimes \widehat{R_{\mathfrak{m}}}$ is a holonomic $D$-module.

As $(R / P)_{\mathfrak{m}}$ is regular local we may assume that $P R_{\mathfrak{m}}=\left(Z_{1}, \ldots, Z_{g}\right)$. Note $n \geq g+2$. In particular we have that $P \widehat{R_{\mathfrak{m}}}$ is a prime ideal in $\widehat{R_{\mathfrak{m}}}$. Notice $V$ is $P \widehat{R_{\mathfrak{m}}}$-torsion. Furthermore Ass $V=\left\{P \widehat{R_{\mathfrak{m}}}\right\}$. Using Proposition 2.6 we get that there exists $h \in \widehat{R_{\mathfrak{m}}} \backslash P \widehat{R_{\mathfrak{m}}}$ such that $\operatorname{Hom}\left(\widehat{R_{\mathfrak{m}}} / P \widehat{R_{\mathfrak{m}}}, V\right)_{h}$ is a finitely generated $\left(\widehat{R_{\mathfrak{m}}}\right)_{h}$-module. Notice $\operatorname{Hom}_{R_{\mathfrak{m}}}\left(R_{\mathfrak{m}} / P R_{\mathfrak{m}}, E(R / P)_{\mathfrak{m}}\right)=k(P)$ where $k(P)$ is the quotient field of $R_{\mathfrak{m}} / P R_{\mathfrak{m}}$. It follows that

$$
\operatorname{Hom}\left(\widehat{R_{\mathfrak{m}}} / P \widehat{R_{\mathfrak{m}}}, V\right)=\operatorname{Hom}_{R_{\mathfrak{m}}}\left(R_{\mathfrak{m}} / P R_{\mathfrak{m}}, E(R / P)_{\mathfrak{m}}\right) \otimes \widehat{R_{\mathfrak{m}}}=k(P) \otimes \widehat{R_{\mathfrak{m}}} .
$$

For $\lambda \in K$ let $\mathfrak{q}_{\lambda}=\left(Z_{1}, \ldots, Z_{g}, Z_{g+1}+\lambda Z_{g+2}\right)$. Clearly $\mathfrak{q}_{\lambda}$ is a prime ideal of height $g+1$ in $R_{\mathfrak{m}}$ containing $P R_{\mathfrak{m}}$. Furthermore we have that $\mathfrak{q}_{\lambda} \widehat{R_{\mathfrak{m}}}$ is a prime ideal in $\widehat{R_{\mathfrak{m}}}$. If $\lambda_{1} \neq \lambda_{2}$ then it is easy to show that $\mathfrak{q}_{\lambda_{1}} \neq \mathfrak{q}_{\lambda_{2}}$. Now consider $\bar{h}$, the image of $h$ in $\widehat{R_{\mathfrak{m}}} / P \widehat{R_{\mathfrak{m}}}$. By considering a primary decomposition of $(\bar{h})$ it follows that infinitely many $\mathfrak{q}_{\lambda} \widehat{R_{\mathfrak{m}}}$ do not contain $h$. Choose one such $\lambda$. Thus we have that $\operatorname{Hom}\left(\widehat{R_{\mathfrak{m}}} / P \widehat{R_{\mathfrak{m}}}, V\right)_{\mathfrak{q}_{\lambda} \widehat{R_{\mathfrak{m}}}}$ is a finitely generated $\left(\widehat{R_{\mathfrak{m}}}\right)_{\mathfrak{q}_{\lambda} \widehat{R_{\mathfrak{m}}}}$-module. Notice we have a flat local map $\left(R_{\mathfrak{m}}\right)_{\mathfrak{q}_{\lambda}} \rightarrow\left(\widehat{R_{\mathfrak{m}}}\right)_{\mathfrak{q}_{\lambda} \widehat{R_{\mathfrak{m}}}}$. Furthermore note that

$$
\begin{aligned}
\operatorname{Hom}\left(\widehat{R_{\mathfrak{m}}} / P \widehat{R_{\mathfrak{m}}}, V\right)_{\mathfrak{q}_{\lambda} \widehat{R_{\mathfrak{m}}}} & =k(P) \otimes_{R_{\mathfrak{m}}} \widehat{R_{\mathfrak{m}}} \otimes_{\widehat{R_{\mathfrak{m}}}}\left(\widehat{R_{\mathfrak{m}}}\right)_{\mathfrak{q}_{\lambda} \widehat{R_{\mathfrak{m}}}}, \\
& =k(P) \otimes_{R_{\mathfrak{m}}}\left(\widehat{R_{\mathfrak{m}}}\right)_{\mathfrak{q}_{\lambda} \widehat{R_{\mathfrak{m}}}}, \\
& =k(P) \otimes_{R_{\mathfrak{m}}}\left(R_{\mathfrak{m}}\right)_{\mathfrak{q}_{\lambda}} \otimes_{\left(R_{\mathfrak{m}}\right)_{\mathfrak{q}_{\lambda}}}\left(\widehat{R_{\mathfrak{m}}}\right)_{\mathfrak{q}_{\lambda} \widehat{R_{\mathfrak{m}}}}, \\
& =k(P) \otimes_{\left(R_{\mathfrak{m}}\right)_{\mathfrak{q}_{\lambda}}}\left(\widehat{R_{\mathfrak{m}}}\right)_{\mathfrak{q}_{\lambda} \widehat{R_{\mathfrak{m}}}} .
\end{aligned}
$$

In the last equation we have used that $k(P)_{\mathfrak{q}_{\lambda}}=k(P)$. By Proposition 3.3 we get that $k(P)$ is a finitely generated $\left(R_{\mathfrak{m}}\right)_{\mathfrak{q}_{\lambda}}$-module. This is a contradiction as $P\left(R_{\mathfrak{m}}\right)_{\mathfrak{q}_{\lambda}}$ is a non-maximal prime ideal in $\left(R_{\mathfrak{m}}\right)_{\mathfrak{q}_{\lambda}}$.

We need the following result in the proof of Lemma 3.2

Proposition 3.3. Let $\phi: A \rightarrow B$ be a flat local map of Noetherian local rings. Let $L$ be an $A$-module. Then $L$ is finitely generated as a $A$-module if and only if $L \otimes_{A} B$ is finitely generated as a B-module.

Proof. If $L$ is finitely generated as a $A$-module then clearly $L \otimes_{A} B$ is finitely $B$ module. Suppose now that $L$ is not a finitely generated $A$-module. Let

$$
L_{1} \varsubsetneqq L_{2} \varsubsetneqq \cdots \varsubsetneqq L_{n} \varsubsetneqq L_{n+1} \varsubsetneqq \cdots
$$

be a strictly ascending chain of submodules in $L$. By faithful flatness we have that

$$
L_{1} \otimes B \varsubsetneqq L_{2} \otimes B \varsubsetneqq \cdots \varsubsetneqq L_{n} \otimes B \varsubsetneqq L_{n+1} \otimes B \varsubsetneqq \cdots
$$


is a strictly ascending chain of submodules of $L \otimes B$. It follows that $L \otimes B$ is not finitely generated.

\section{Proof of the Theorem 1.1}

In this section we prove our main result. We need the following easily proved fact.

Proposition 4.1. Let $A$ be a Noetherian ring and let $T$ be an A-module. Let $f \in A$. Then the natural map

$$
\eta: T \rightarrow T_{f} \text { is injective if and only if } f \notin \bigcup_{P \in \operatorname{Ass} T} P .
$$

We now give

Proof of Theorem 1.1. Set $M=\mathcal{T}(R)$. We prove that if $P$ is a prime ideal in $R$ and not maximal then $\mu_{c}(P, M)=0$. Notice $\mu_{c}(P, M)=\mu_{0}\left(P, H_{P}^{c}(M)\right)$, see [5, 1.4, 3.4(b)]. We consider two cases.

Case 1: height $P=n-1$.

Suppose if possible $\mu_{0}\left(P, H_{P}^{c}(M)\right) \neq 0$. Notice then $P$ is a minimal prime of $H_{P}^{c}(M)$. So if $\mathfrak{q} \in \operatorname{Ass}_{R} H_{P}^{c}(M)$ and $\mathfrak{q} \neq P$ then $\mathfrak{q}$ is a maximal ideal of $R$. In this case $\Gamma_{\mathfrak{q}}\left(H_{P}^{c}(M)\right)=E(R / \mathfrak{q})^{r}$ for some $r>0$. Since Ass $H_{P}^{c}(M)$ is a finite set we can write $H_{P}^{c}(M)=L \oplus I$ as $R$-modules where $\operatorname{Ass}_{R} L=\{P\}$ and $I=$ $E\left(R / \mathfrak{m}_{1}\right)^{r_{1}} \oplus E\left(R / \mathfrak{m}_{2}\right)^{r_{2}} \oplus \cdots \oplus E\left(R / \mathfrak{m}_{s}\right)^{r_{s}}$ for some maximal ideals $\mathfrak{m}_{1}, \ldots, \mathfrak{m}_{s}$ and finite numbers $r_{1}, \ldots, r_{s}$. Thus $I$ is an injective $R$-module. Also note that both $L$ and $I$ are $P$-torsion. Further note that $I=\Gamma_{\mathfrak{m}_{1} \mathfrak{m}_{2} \cdots \mathfrak{m}_{s}}\left(H_{P}^{c}(M)\right)$ is a $A_{n}(K)$ submodule of $H_{P}^{c}(M)$ and so $L \cong H_{P}^{c}(M) / I$ is a holonomic $A_{n}(K)$-module.

Let $f \in R \backslash P$. Recall injdim $M=c$. We have an exact sequence

$$
H_{(P, f)}^{c}(M) \rightarrow H_{P}^{c}(M) \rightarrow H_{P}^{c}(M)_{f} \rightarrow H_{(P, f)}^{c+1}(M)=0 .
$$

Thus the natural map $\eta: L \rightarrow L_{f}$ is surjective. As $f \notin P$ and Ass $L=\{P\}$ we get that $\eta$ is also injective. Thus $L=L_{f}$ for every $f \in R \backslash P$. It follows that $L=L_{P}$. Also note that $L_{P}=H_{P}^{c}(M)_{P}$. By [5, 3.4(b)], $L_{P}=E(R / P)^{l}$ for some finite $l>0$. Thus we have that $E(R / P)$ is a holonomic $A_{n}(K)$-module. By 3.1 this is a contradiction.

Case 2: height $P \leq n-2$.

Suppose if possible $\mu_{0}\left(P, H_{P}^{c}(M)\right) \neq 0$. Let Ass $H_{P}^{c}(M)=\left\{P, Q_{1}, \ldots Q_{c}\right\}$ where $Q_{i} \neq P$. As $H_{P}^{c}(M)$ is $P$-torsion we have that $Q_{i} \supsetneq P$ for all $i$. Let $f_{i} \in Q_{i} \backslash P$. Put $f=f_{1} \cdots f_{c}$. By Theorem 2.8 (4) there exists a maximal ideal $\mathfrak{m}$ of $R$ such that $f \notin$ $\mathfrak{m}$ and $(R / P)_{\mathfrak{m}}$ is regular local. Localize at $\mathfrak{m}$. Notice $\operatorname{Ass}_{R_{\mathfrak{m}}} H_{P}^{c}(M)_{\mathfrak{m}}=\left\{P R_{\mathfrak{m}}\right\}$. Let $g \in R_{\mathfrak{m}} \backslash P R_{\mathfrak{m}}$. Notice $\operatorname{injdim}_{R_{\mathfrak{m}}} M_{\mathfrak{m}} \leq c$. So we have an exact sequence

$$
H_{\left(P R_{\mathfrak{m}}, g\right)}^{c}\left(M_{\mathfrak{m}}\right) \rightarrow H_{P R_{\mathfrak{m}}}^{c}\left(M_{\mathfrak{m}}\right) \rightarrow H_{P R_{\mathfrak{m}}}^{c}\left(M_{\mathfrak{m}}\right)_{g} \rightarrow H_{\left(P R_{\mathfrak{m}}, g\right)}^{c+1}\left(M_{\mathfrak{m}}\right)=0 .
$$

Thus the natural map $\eta: H_{P R_{\mathfrak{m}}}^{c}\left(M_{\mathfrak{m}}\right) \rightarrow H_{P R_{\mathfrak{m}}}^{c}\left(M_{\mathfrak{m}}\right)_{g}$ is surjective. By Lemma 4.1 it is also injective as Ass $H_{P R_{\mathfrak{m}}}^{c}\left(M_{\mathfrak{m}}\right)=\left\{P R_{\mathfrak{m}}\right\}$. It follows that $H_{P R_{\mathfrak{m}}}^{c}\left(M_{\mathfrak{m}}\right)=$ $H_{P R_{\mathfrak{m}}}^{c}\left(M_{\mathfrak{m}}\right)_{g}$. So $H_{P R_{\mathfrak{m}}}^{c}\left(M_{\mathfrak{m}}\right)=H_{P R_{\mathfrak{m}}}^{c}\left(M_{\mathfrak{m}}\right)_{P R_{\mathfrak{m}}}$. By [5, 1.4, 3.4(b)], we get that $H_{P R_{\mathfrak{m}}}^{c}\left(M_{\mathfrak{m}}\right)_{P} \cong E\left(R_{\mathfrak{m}} / P R_{\mathfrak{m}}\right)^{s}$ for some finite $s>0$. By 2.2 there exist a Lyubeznik functor $\mathcal{T}^{\prime}$ on $\operatorname{Mod}\left(R_{\mathfrak{m}}\right)$ with $\mathcal{T}^{\prime}\left(R_{\mathfrak{m}}\right)=\mathcal{T}(R) \otimes R_{\mathfrak{m}}=M_{\mathfrak{m}}$. Observe that $\mathcal{G}=$ $H_{P R_{\mathfrak{m}}}^{c} \circ \mathcal{T}^{\prime}$ is a Lyubeznik functor on $R_{\mathfrak{m}}$. We have $\mathcal{G}\left(R_{\mathfrak{m}}\right)=E\left(R_{\mathfrak{m}} / P R_{\mathfrak{m}}\right)^{s}$. This contradicts Lemma 3.2 . 


\section{Proof of Proposition 1.2}

In this section we prove Proposition 1.2. Throughout $K$ is an algebraically closed field of characteristic zero. Let $R=K\left[X_{1}, \ldots, X_{n}\right]$ and let $A_{n}(K)$ be the $n^{\text {th }}$-Weyl algebra over $K$. We use notions developed in [2, Chapter 1], in particular we use the notion of Bernstein filtration of $A_{n}(K)$, good filtration, multiplicity and dimension of a finitely generated $A_{n}(K)$-module. We will use the fact that for any holonmic module $M$ we have $\ell(M) \leq e(M)$; here $\ell(M)$ denotes the length of $M$ as an $A_{n}(K)$-module and $e(M)$ denotes its multiplicity.

The following result is well-known. So we just sketch an argument.

Proposition 5.1. Let $\mathfrak{m}$ be a maximal ideal of $R$. Then $e(E(R / \mathfrak{m}))=1$. In particular $E(R / \mathfrak{m})$ is a simple $A_{n}(K)$-module.

Proof. (Sketch) As $K$ is algebraically closed $\mathfrak{m}=\left(X_{1}-a_{1}, \ldots, X_{n}-a_{n}\right)$ for some $a_{1}, \ldots, a_{n} \in K$. After a change of variables we may assume $a_{1}=\cdots=a_{n}=0$. Note $E(R / \mathfrak{m})=K\left[\partial_{1}, \ldots, \partial_{n}\right]$. The obvious filtration on $E(R / \mathfrak{m})$ is compatible with the Bernstein filtration and is good. So $e(E(R / \mathfrak{m}))=1$.

5.2. Let $M$ be a holonomic $A_{n}(K)$-module. Let $f \in R$ be a polynomial of degree $d$. Then by proof of Theorem 5.19 in Chapter 1 of [2] we have

$$
e\left(M_{f}\right) \leq e(M)(1+\operatorname{deg} f)^{n} .
$$

We now give

Proof of Proposition 1.2. Set $M=\mathcal{T}(R)$. Let $\mathfrak{m}=\left(X_{1}-a_{1}, \ldots, X_{n}-a_{n}\right)$ be a maximal ideal of $R$. Fix $i$ with $0 \leq i \leq c$. Notice $\mu_{i}(\mathfrak{m}, M)=\mu_{0}\left(\mathfrak{m}, H_{\mathfrak{m}}^{i}(M)\right)$, see [5, 1.4, 3.4(b)]. If $H_{\mathfrak{m}}^{i}(M)=E(R / \mathfrak{m})^{r_{i}}$ then $\mu_{i}(\mathfrak{m}, M)=r_{i}=\ell\left(H_{\mathfrak{m}}^{i}(M)\right)$. To compute $H_{\mathfrak{m}}^{i}(M)$ we use the Čech-complex:

$$
\mathbf{C}: 0 \rightarrow M \rightarrow \bigoplus_{j=1}^{n} M_{\left(X_{j}-a_{j}\right)} \rightarrow \cdots \rightarrow M_{\left(X_{1}-a_{1}\right) \cdots\left(X_{n}-a_{n}\right)} \rightarrow 0 .
$$

In particular we have that $\ell\left(H_{\mathfrak{m}}^{i}(M)\right) \leq \ell\left(\mathbf{C}^{i}\right)$. Notice $C^{i}$ has $\left(\begin{array}{c}n \\ i\end{array}\right)$ copies of modules of the form $M_{f}$ were $f$ is a product of $i$ distinct polynomials among $X_{1}-a_{1}, \cdots, X_{n}-a_{n}$. In particular $\operatorname{deg} f=i$. So by 5.2 we have $e\left(M_{f}\right) \leq$ $e(M)(1+i)^{n}$. Thus

$$
r_{i} \leq e\left(\mathbf{C}^{i}\right) \leq\left(\begin{array}{c}
n \\
i
\end{array}\right) e(M)(1+i)^{n}
$$

Remark 5.3. If $K_{p}$ is an algebraically closed field of characteristic $p$ and $S=$ $K_{p}\left[X_{1}, \ldots, X_{n}\right]$ then Proposition 1.2 holds for functors of the form

$$
\mathcal{G}(-)=H_{I_{1}}^{i_{1}}\left(H_{I_{2}}^{i_{2}}\left(\cdots\left(H_{I_{r}}^{i_{r}}(-)\right) \cdots\right) .\right.
$$

The point is that $\mathcal{G}(R)$ is holonomic $D$-module where $D$ is the ring of $K_{p}$-linear differential operators over $S$. Here we use the notion of holonomicity by V. Bavula [1. In this case the bound $\ell\left(M_{f}\right) \leq n ! \ell(M)(1+\operatorname{deg} f)^{n}$ holds, see [7, Proof of 3.6]. The proof then follows by the same argument as before. 


\section{REFERENCES}

[1] V. Bavula, Dimension, multiplicity, holonomic modules, and an analogue of the inequality of Bernstein for rings of differential operators in prime characteristic, Representation Theory, Electron. J. AMS 13 (2009) 182-227.

[2] J. -E. Björk, Rings of Differential Operators, Amsterdam, North-Holland, 1979.

[3] W. Bruns and J. Herzog, Cohen-Macaulay Rings, revised edition, Cambridge Stud. Adv. Math., vol. 39, Cambridge University Press, Cambridge, (1998).

[4] C. Huneke and R. Sharp, Bass Numbers of Local Cohomology Modules, AMS Transactions 339 (1993), 765-779.

[5] G. Lyubeznik, Finiteness Properties of Local Cohomology Modules (an Application of Dmodules to Commutative Algebra), Inv. Math. 113 (1993), 41-55.

[6] _ F-modules: applications to local cohomology and D-modules in characteristic $p_{\dot{\zeta}} 0$, J. Reine Angew. Math. 491 (1997), 65-130.

[7] _ A characteristic-free proof of a basic result on D-modules, J. Pure Appl. Algebra 215 (2011), no. 8, 2019-2023.

[8] H. Matsumura, Commutative ring theory, second ed., Cambridge Studies in Advanced Mathematics, vol. 8, Cambridge University Press, Cambridge, 1989.

Department of Mathematics, Indian Institute of Technology Bombay, Powai, Mumbai 400 076, INDIA

E-mail address: tputhen@math.iitb.ac.in 